\title{
PROGRAMA ALMIDA. PROGRAMA DE MEJORA DE LAS COMPETENCIAS SOCIOCOGNITIVAS EN PERSONAS CON CAPACIDADES DIFERENTES ALMIDA PROGRAM. IMPROVEMENT PROGRAM OF SOCIAL - COGNITIVE SKILLS IN PEOPLE WITH DIFFERENT ABILITIES
}

\author{
Ángel Luis GonZÁlez Olivares ${ }^{1}$ \\ Rosario CRUZ MARTín-SERRANO ${ }^{2}$ \\ ${ }^{1}$ Facultad de Educación. UNED \\ ${ }^{2}$ Grupo de Integración Almida, Ciudad Real \\ algonzalez@edu.uned.es
}

Cómo referenciar este artículo/How to reference this article:

González-Olivares, A. L. y Martín-Serrano, R. C. (2012). Programa Almida. Programa de Mejora de las Competencias Sociocognitivas en Personas con Capacidades Diferentes. Acción Psicológica, 9(2), 59-76 doi: http://dx.doi.org/10.5944/ap.9.2.4105

\section{Resumen}

El Programa de Mejora de las Competencias Sociocognitivas en Personas con Capacidades Diferentes es un conjunto de actuaciones de orientación social y laboral y asesoramiento para la consecución de competencias sociales, intelectuales y cognitivas, con la finalidad de mejora de la autonomía personal, la responsabilidad del proceso de aprendizaje personal y profesional, y las condiciones que favorecen la adquisición de competencias de afrontar retos y desafíos de la vida de cada persona con discapacidad. Este Programa está identificado con un enfoque ecológico. Enfoque que defiende que la acción orientadora ha de plantearse desde las características y demandas del ambiente de cada persona, así como de las habilidades y destrezas funcionales necesarias para la adaptación del sujeto. Además realiza un diagnóstico vocacional planteado desde una concepción holística y ecológica, reconociendo al sujeto como un todo, no como una suma de habilidades e inhabilidades, y no separado de los contextos naturales donde vive y encuentra significado su forma de ser. El presente estudio tiene como objetivo principal comprobar, desde el punto de vista científico, la eficiencia del Programa Almida como un Programa de Mejora de las Competencias Sociocognitivas en Personas con Capacidades Diferentes.

Palabras clave: competencias sociocognitivas, capacidades diferentes, orientación, intervención psicopedagógica, enfoque ecológico.

Abstract
Improvement Program of social-cognitive Skills
in People with Different Abilities is a set of so-
cial activities and occupational guidance and
counseling skills to achieve social, intellectual
and cognitive, with the aim of improving per-
sonal autonomy, responsibility for the process
personal and professional learning and the
conditions that favor the acquisition of skills


to address issues and challenges for the life of every person with a disability. This program is identified with an ecological approach. Approach advocates that action must arise from the characteristics and environmental demands of each person and the skills and functional skills necessary to adapt the subject. Besides vocational raised a diagnosis from a holistic and ecological, recognizing the subject as a whole, not as a sum of abilities and disabilities, and not separated from the natural settings where they live and how they are meant to be. This study's main objective is to verify, from the scientific standpoint, the efficiency of Almida Program as a Program Improvement in social-cognitive Skills Disabled People.

Keywords: social-cognitive skills, disabilities, counseling, intervention, educational psychology, ecological approach.

\section{Introducción}

El Programa Almida es un conjunto de procesos dirigidos a personas con discapacidad necesitadas de orientación, entendida ésta como una intervención psicopedagógica que trata de ayudarles a conseguir los conocimientos y habilidades que necesitan.

Las personas con capacidades diferentes reciben una instrucción sistemática, gradual y diferenciada dirigida a la consecución de competencias sociales, intelectuales y cognitivas, que pretenden mejorar la autonomía personal, la responsabilidad sobre el proceso de aprendizaje, personal y profesional, y las condiciones que favorecen la adquisición de competencias para afrontar los retos y desafíos que conlleva la vida.

El presente estudio tiene como objetivo principal comprobar, desde el punto de vista científico, la eficiencia del Programa Almida como un Programa de Mejora de las Competencias Sociocognitivas en Personas con Capacidades Diferentes.

\section{Programa de Mejora de las Competencias Sociocognitivas en personas con Capacidades Diferentes (Programa Almida)}

El Programa de Mejora de las Competencias Sociocognitivas es un conjunto de actuaciones de orientación social y laboral y asesoramientos para la consecución de competencias sociales, intelectuales y cognitivas, que pretenden mejorar la autonomía personal, la responsabilidad sobre el proceso de aprendizaje, personal y profesional y las condiciones que favorezcan la adquisición de competencias para afrontar los retos y desafíos que conlleva la vida de cada persona con discapacidad que es intervenida por el Departamento de Orientación del Grupo de Integración Almida.

El Grupo de Integración Almida es un Centro Especial de Empleo de la Junta de Comunidades de Castilla La Mancha, cuyo objetivo principal es el proporcionar a los trabajadores con discapacidad la realización de un trabajo productivo y remunerado, adecuado a sus características personales, facilitándoles una integración social y laboral en la sociedad.

El Programa Almida se inspira principalmente en el nuevo paradigma educativo que recoge las líneas centrales de la Psicología Cognitiva, especialmente las relacionadas con las nuevas interpretaciones de la inteligencia humana. En personas con capacidades diferentes la orientación es una intervención psicopedagógica que tiene como finalidad la integración social y laboral.

\section{Procesos Cognitivos en el Programa Almida}

Los Procesos del Programa Almida suponen un cambio para la persona, en varios sentidos: mejora de competencias para afrontar sus expectativas, mejora en la percepción misma (autoconcepto) y mejora en la autovaloración que se hace de sí misma (autoestima). En este camino, el concepto de competencia se centra en la capacidad de la persona para situarse favorablemente ante las oportunidades de empleo y de las diferentes situaciones sociales. 


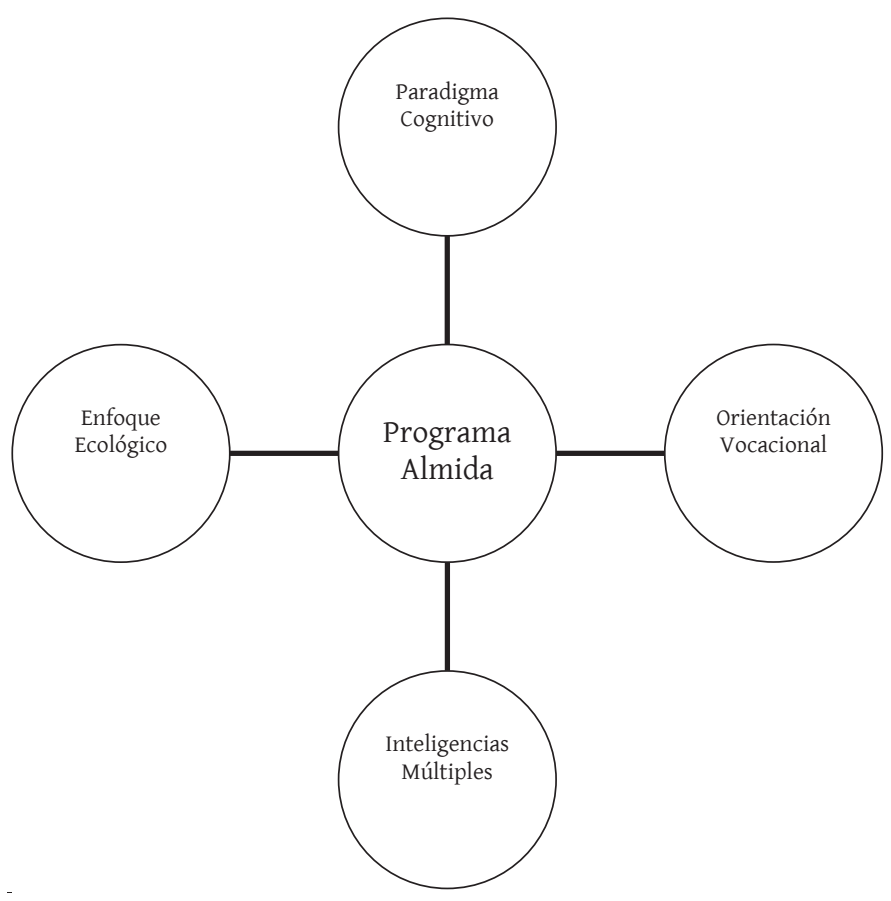

Figura 1. Fundamentos teóricos y científicos del programa de mejora de las competencias sociocognitivas de personas con capacidades diferentes. Fuente: elaboración propia

Tabla 1

\section{Programa Almida}

Objetivos: Mejorar las competencias sociocognitivas en las personas con capacidades diferentes.

Habilidades: Consecución de competencias sociales, intelectuales y cognitivas que mejoren la autonomía personal, la responsabilidad sobre el proceso de aprendizaje, personal y profesional, y las condiciones que favorezcan la adquisición de competencias para afrontar retos y desafíos de la vida.

Pilares Básicos: Paradigma Cognitivo. Orientación Vocacional. Enfoque Ecológico. Inteligencias Múltiples.

Investigación: La investigación se ha hecho sobre la mejora de las competencias sociocognitivas en las personas con capacidades diferentes; mejora de la autonomía personal, la responsabilidad sobre el proceso de aprendizaje y la adquisición de nuevas competencias.

Población: Personas con discapacidad con certificado oficial de los Centros Bases de la Consejería de Salud y Bienestar Social de Castilla La Mancha, en edad laboral (16 a 64 años).

Forma de trabajo: Proceso de Orientación para la competencia sociocognitiva: análisis y diagnóstico inicial, programa de ajustes personales y planificación del contexto marco, intervención en el contexto marco, valoración e informe de competencia sociocognitiva.

Tiempo: mínimo 1 año, secuencializado en distintas fases temporales y estructurado en 28 sesiones de tutoría y orientación de aproximadamente $35 \mathrm{~min}$. de duración. 
Partimos de que «la mayoría de las personas tienen un potencial como para desarrollar unas habilidades de pensamiento muchísimo más eficaces que las que tienen" [Nickerson y otros (1985), pág. 83]. Y que "no hay que perder de vista el hecho de que lo que importa realmente en el mundo no es el nivel de inteligencia que tengamos, sino lo que seamos capaces de obtener con esa inteligencia» (Sternberg 1997, pág. 131).

Diferenciamos dentro de la competencia para el empleo, dos dimensiones de la Empleabilidad: la interna, basada en la persona, conjugada con un conjunto de factores (cualificaciones profesionales, capacidades, habilidades, aptitudes y actitudes, autoconocimiento, reconocimiento de oportunidades, desarrollo profesional,...) y la dimensión externa, que relaciona a la persona con un mercado de trabajo en constante evolución y transformación (Ceniceros y Oteo, 2003; Cáritas, 1999).

El Programa Almida pone de manifiesto la importancia que tiene reforzar la confianza en sí mismo de los participantes, afianzar su sentimiento de pertenencia a un grupo y mejorar la capacidad de identificar y resolver problemas. También pretende no sólo mejorar las capacidades personales, sino también propiciar la integración social y laboral de las personas para que puedan identificar los obstáculos presentes en el mercado de trabajo y generar estrategias individuales y colectivas para enfrentarlos.

\section{Fases y Procesos del Programa de Mejora de las Competencias Sociocognitivas en Personas con Capacidades Diferentes}

Aunque el Programa Almida se desarrolla en un conjunto estandarizado de procesos y procedimientos técnicos, que de manera simplificada, se describe en un conjunto de fases continuadas:

1. ${ }^{\text {a }}$ Análisis y Diagnóstico Inicial (Temporalidad 4 sesiones): por medio de una entrevista individual, se diagnostica la situación de la persona, se efectúa una valoración de su nivel de Empleabilidad y su nivel de Competencia Social y se le propone información preliminar que le ayude a decidir respecto a su itinerario de inserción. Se realiza también un diagnóstico orientativo de las capacidades múltiples y se aplica los instrumentos y pruebas que descubren o detectan el nivel de depresión, un amplio rango de problemas psicopatológicos, el bienestar psicológico, el autoconcepto y las estrategias de aprendizaje de cada persona participante antes de comenzar el tratamiento. Se recaban, además de los datos personales, características del perfil del trabajador, formación académica y la información de la experiencia laboral.

2. ${ }^{\text {a }}$ Programa de Ajustes Personales y Planificación del Contexto Marco: tomando como punto de partida las vivencias de cada persona, de sus necesidades, conflictos y motivaciones, de su entorno y de sus propios recursos, se concreta su compromiso con el proceso de cambio y se fijan unos objetivos y unas acciones determinadas para lograrlos, estableciendo en conjunto una Planificación del Contexto Marco, contexto donde se desarrollarán las competencias sociocognitivas. En esta fase se desarrollan tres acciones complementarias:

Acción 1: Aceptación de nuestro Autoconocimiento y Planificación del Contexto Marco. (Temporalidad: 3 sesiones). Las personas con capacidades diferentes, principalmente debido a sus experiencias de vida, han desarrollado una menor autoestima de sí mismas, lo que influye de forma importante en la posición y movilidad en el mercado laboral. El orientador interviene, en una primera parte, analizando los resultados de la Fase de Análisis y Diagnóstico Inicial relacionados con el nivel de depresión (originada por sus discapacidades, enfermedades, situaciones personales y sociales, etc.), los problemas psicopatológicos (Somatizaciones, Obsesiones y compulsiones, Sensibilidad interpersonal,...), el bienestar psicológico y el autoconcepto, para posteriormente actuar individualmente en su mejora, y realizar un diagnóstico vocacional que ayude a la Planificación del Contexto Marco.

El análisis de la experiencia previa en las situaciones de la vida y las oportunidades laborales buscan promover la reflexión colectiva sobre los obstáculos más frecuentes que en- 
frentan las personas en el ámbito laboral y los hitos más críticos que enfrentan en el transcurso de su historia laboral.

Acción 2: Herencia y Capital de Pertenencia y Cooperación. (Temporalidad: 2 sesiones). Se invita a los participantes a valorar lo que es pertenecer a algún colectivo o grupo. Y se indaga cuáles son los grupos de pertenencia de cada cual, distinguiendo los derechos y responsabilidades que conlleva la pertenencia y destacan los vínculos y las redes sociales. El orientador ayuda a mejorar la sensibilidad interpersonal, la capacidad de mantener relaciones positivas con otras personas y el autoconcepto familiar y social, tomando como base el enfoque ecológico (García Pastor y Alvarez Rojo, 1995).

Acción 3: Aprender a buscar soluciones. (Temporalidad: 4 sesiones). La idea principal es ayudar a los participantes a reconocer y buscar soluciones. Se sugiere que los participantes sean capaces de identificar los distintos factores que configuran una situación problemática, considerándose como un factor más en la constitución de situaciones problemáticas y de gran importancia para la solución de problemas. La atribución de control parece aquí un punto central, en especial en el sentido que las personas comprendan que pueden enfrentarse a los problemas y que es posible adquirir a través del aprendizaje maneras más eficientes de enfrentarlos y resolverlos.

Destacamos las peculiaridades de la orientación en al Programa Almida donde el orientador es: a) Un facilitador de aprendizajes: las personas con capacidades diferentes tienen que aprender, y aprender de manera significativa. La organización y diseño de la formación profesional de este colectivo considera la importante utilización del andamiaje, los conocimientos y experiencias previas, los aprendizajes funcionales y cooperativos, como también la transferencia del aprendizaje; b) Un mediador del aprendizaje: la persona con capacidades diferentes es el núcleo esencial del proceso de formación. El orientador identifica los procesos de aprendizaje (sensibilización, atención, adquisición, personalización y control, recuperación, aplicación y evaluación) para desarrollarlos y mejorarlos. Y facilita las estrategias de aprendizaje (sensibili- zación, atención, adquisición, personalización y control, recuperación, transfer y evaluación).

\section{3. ${ }^{a}$ Intervención en el Contexto Marco.} (Temporalidad: mínimo 10 sesiones). Aplicación de actuaciones de intervención para la Mejora de las Competencias Sociocognitivas y la Empleabilidad de las Personas con Capacidades Diferentes: a) Entrevistas específicas, tutorías, información, orientación y seguimientos personalizado, incluyendo acciones de desarrollo de aspectos personales que favorezca la ocupación, b) Programas de Formación Profesional y Ocupacional para proporcionar habilidades, técnicas y recursos para desempeñarse profesionalmente, mediante la cualificación, recualificación o actualización de competencias profesionales, con la acreditación por medio de las certificaciones correspondientes (formación profesional reglada, formación ocupacional, talleres pre laborales, cursos de formación a la carta,...); c) Bolsa de Empleo: servicio basado en el conocimiento de las personas demandantes de empleo y de sus características formativas y profesionales, en el que se atiende a las ofertas de empleo perfiles adecuados a las necesidades y requisitos, contribuyendo a la incorporación laboral de personas con capacidades diferentes al acceso al empleo, d) Programa de Atención y de Apoyo al Autoempleo, encaminado a suscitar el desarrollo de emprendedores y dar seguimiento a iniciativas de autoempleo. Son actuaciones como la provisión de información (trámites y requisitos legales, fiscalidad, ayudas a la creación de empleo, etc.) formación básica en gestión empresarial, talleres de autoempleo, elaboración de materiales y guías, apoyo en la elaboración del plan de empresa, realización de estudios de viabilidad, etc.; e) Modelos de Empleabilidad: 1) Empleo con Inicio de Actividad: programa mixto de formación y empleo. Preparación para el trabajo con la combinación de formación teórica especializada y la práctica laboral; 2) Empleo con Adaptación y Supervisión: sistema de soporte técnico e individualizado que ayuda a personas con discapacidad a encontrar o mantener un trabajo remunerado mediante la realización de un servicio en una empresa ordinaria, ya sea pública o privada; 3) Empleo con Apoyo y Ocupación Protegida: fórmula de empleo protegido para conseguir la 
mayor integración de las personas con capacidades diferentes.

El desarrollo de la Fase de Intervención no implica que todas las personas deben recorrer todas las subetapas y tipologías descritas, sino que de manera particular, y atendiendo a las características personales de cada caso, el usuario/a atendido/a seguirá un itinerario diseñado y adaptado a su perfil personal.

\section{4. ${ }^{\text {a }}$ Seguimiento y Apoyo en el Contexto} Marco. (Temporalidad: depende de cada caso, mínimo 5 sesiones). Durante el itinerario de inserción, una vez incorporado a un puesto de trabajo, se realizan entrevistas específicas y tutorías, donde se informa, orienta y realizan intervenciones personalizadas, incluyendo acciones de desarrollo de aspectos personales que favorezca la ocupación y las competencias sociocognitivas, con el fin de detectar dificultades que afectan negativamente al mantenimiento del empleo e intentar eliminarlas.

\section{5. ${ }^{a}$ Informe de Competencia Sociocogniti-} va. Valoración del proceso, evaluando los conocimientos y destrezas adquiridos, los cambios en la actitud experimentados por la persona usuaria, nuevo perfil de Competencia Sociocognitiva y perspectivas de futuro.

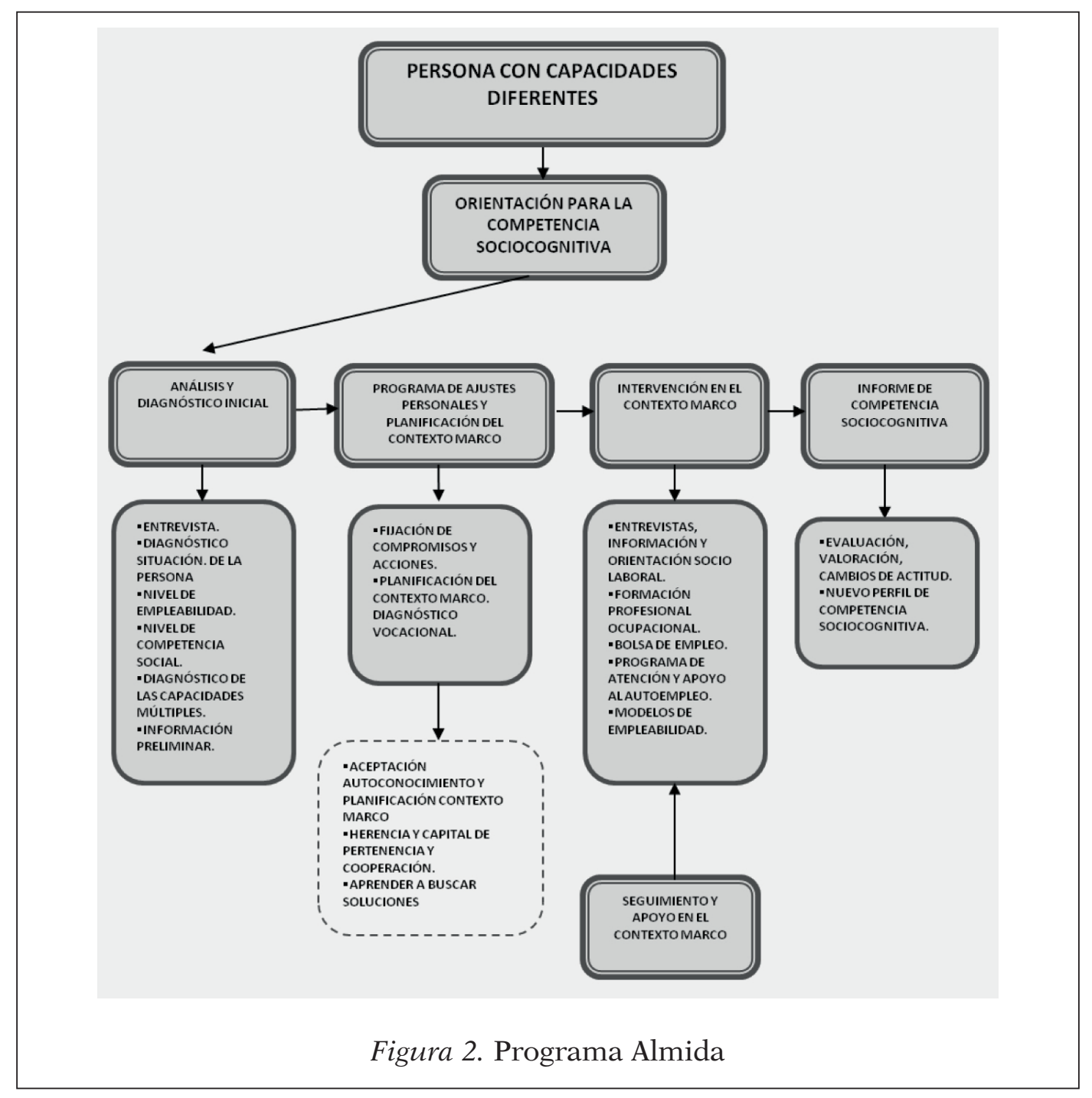




\section{Objetivos de la investigación}

El porqué del presente estudio es comprobar la eficiencia del Programa Almida como un Programa de Mejora de las Competencias Sociocognitivas en personas con Capacidades Diferentes, que se desglosa en un objetivo general y varios específicos.

Objetivo General:

- Comprobar, desde el punto de vista científico, la eficiencia del Programa Almida: Programa de Mejora de las Competencias Sociocognitivas en Personas con Capacidades Diferentes, como conjunto de actuaciones de orientación social y laboral y asesoramientos para la consecución de competencias sociales, intelectuales y cognitivas, que pretenden mejorar la autonomía personal, la responsabilidad sobre el proceso de aprendizaje y la adquisición de competencias que ayuden a superar retos y desafíos de la vida.

Para dar respuesta al objetivo general, se plantean los siguientes objetivos específicos:

- Evaluar la implementación del Programa Almida identificando los principales factores de cambio en las personas participantes: a) Evaluar y mejorar los estados emocionales y la gravedad (intensidad sintomática) de la depresión; b) Valorar y mejorar principales de problemas psicopatológicos; c) Optimar el bienestar psicológico; d) Optimizar el autoconcepto de las personas con capacidades diferentes en sus cinco dimensiones: académico-laboral, emocional, familiar, físico y social; e) Perfeccionar las estrategias de aprendizaje.

\section{Hipótesis de la investigación} tesis:

El estudio ha planteado las siguientes hipó-

La aplicación del Programa Almida mejora:

a. el estado emocional de las personas participantes que presenten algunos problemas de carácter depresivo. b. el estado de ánimo de los participantes afectados de problemas psicopatológicos.

c. el bienestar psicológico de las personas participantes.

d. el autoconcepto de las personas participantes.

e. el nivel de estrategias de aprendizaje de las personas participantes.

\section{Método}

Tanto el proceso de investigación como el de intervención acontecen en interacción permanente. Intervención e investigación pueden ser consideradas como posiciones que se adopten en el desarrollo de la práctica profesional, de manera alternativa.

El enfoque metodológico que se utiliza en esta perspectiva es la metodología cualitativa y también la cuantitativa, en la que conviene destacar que hay una participación plena de la persona en el programa. El Programa coloca al participante en el centro de la actuación. Solamente después de conocer en profundidad su perfil de competencia sociocognitiva y contar con su plena concienciación respecto al proceso que inicia y se responsabiliza, se pueden diseñar y poner en marcha acciones para facilitar la mejora de la autonomía personal, la responsabilidad sobre el proceso de aprendizaje y la adquisición de competencias para afrontar retos y desafíos que la vida conlleva.

En definitiva, esta metodología parte de lo social a lo laboral, porque favorece la incorporación al mundo laboral de los colectivos desfavorecidos. Es también una metodología que se nutre de las fuentes de lo social con la utilización de la técnica de la entrevista individualizada y adaptada a necesidades como elemento fundamental para intervenir con personas cuyas necesidades y carencias van más allá de los puramente laboral. Asimismo parte de conceptos propios de lo social como el desarrollo de itinerario de inserción sociolaboral, de la conceptualización de los mis- 
mos como procesos y de la recuperación del protagonismo creciente de la persona en su camino hacia el empleo y la ciudadanía, como persona con competencias para tener éxito en la vida.

\section{Participantes y muestra}

Mediante una selección aleatoria de las personas con discapacidad reconocidas por el Centro Base de la Consejería de Sanidad y Asuntos Sociales de Castilla La Mancha, en situación de desempleo, inscritos en las Oficinas de Empleo del Servicio Público de Empleo de Castilla La Mancha y participantes de las actividades de los Programas del Centro Especial de Empleo del Grupo de Integración Almida, se ha obtenido una muestra de 71 personas con discapacidad, de una población directa de 725 personas.

La muestra forma parte de un grupo que ha tenido dos momentos de evaluación: pretest y postest.

\section{Descripción de la muestra}

La distribución de los sujetos en función de la muestra con sus características se puede observar en la Tabla siguiente.

Teniendo en cuenta que 3 personas comenzaron el tratamiento pero lo abandonaron, te- nemos una muestra final de 68 sujetos. La edad media es 37,47 años, y ha oscilado entre 19 y 62 años.

\section{Instrumentos}

A los sujetos se le aplicaron los siguientes instrumentos:

a. Inventario de Depresión de Beck. El BDI utilizado en nuestra investigación es un cuestionario autoaplicado de 21 ítems que evalúa un amplio espectro de síntomas depresivos. Se sistematizan 4 alternativas de respuesta para cada ítem, que evalúan la gravedad / intensidad del síntoma y que se presentan igualmente ordenadas de menor a mayor gravedad. El marco temporal hace referencia al momento actual y a la semana previa. Su contenido enfatiza más en el componente cognitivo de la depresión, ya que los síntomas de esta esfera representan en torno al $50 \%$ de la puntuación total del cuestionario, siendo los síntomas de tipo somático / vegetativo el segundo bloque de mayor peso; de los 21 ítems, 15 hacen referencia a síntomas psicológico-cognitivos, y los 6 restantes a síntomas somático-vegetativos. Sus índices psicométricos han sido estudiados de manera casi exhaustiva, mostrando una buena consistencia interna (alfa de Cronbach 0,76 - 0,95).

Tabla 2

Muestra de Investigación

\begin{tabular}{|c|c|c|c|c|c|c|}
\hline $\mathbf{N}$ & Edad media & Sexo & $\mathbf{N}$ & Nivel academico & $\mathbf{N}$ & $\begin{array}{c}\text { Tipo } \\
\text { de discapacidad }\end{array}$ \\
\hline \multirow[t]{7}{*}{68} & 37.47 & 35 Mujeres & 3 & Sin estudios & 47 & Física \\
\hline & & 33 Hombres & 30 & EGB & 4 & Intelectual \\
\hline & & & 7 & FP1 & 4 & Sens. Visual \\
\hline & & & 4 & FP2 & 2 & Sens. Auditiv. \\
\hline & & & 11 & ESO & 11 & Mental \\
\hline & & & 8 & Bachiller & & \\
\hline & & & 5 & Universitarios & & \\
\hline
\end{tabular}


La fiabilidad test oscila alrededor de $r=0,8$, pero su estudio ha presentado dificultades metodológicas, recomendándose en estos casos variaciones a lo largo del día en su administración. Su validez predictiva como instrumento diagnóstico de cribado ha sido recientemente estudiada en nuestro país en una amplia muestra de población general de entre 18 y 64 años de edad, con buen rendimiento: sensibilidad $100 \%$, especificidad $99 \%$, valor predictivo positivo 0.72 , y valor predictivo negativo 1 (punto de corte $\geq 13$ ).

b. Symton Check List. El SCL-90-R es un cuestionario multidimensional autoadministrado. La Persona con Capacidades Diferentes tiene que seleccionar mediante una escala tipo Likert de cinco grados, hasta qué punto se ha sentido molesto, durante los últimos siete días, por una serie de 90 problemas (0 no la ha molestado en absoluto y 4 le ha molestado de manera extrema). El cuestionario explora 9 dimensiones o perfiles psicopatológicos: Somatización, Obsesión Compulsión, Sensibilidad Interpersonal, Depresión, Ansiedad, Ira-Hostilidad, Ansiedad Fóbica, Ideación Paranoide y Psicoticismo. Los coeficientes calculados de fiabilidad par el total de la escala y para cada una de las subescalas han sido superiores a 0.70 alcanzando valores hasta de 0.90. La validez ha sido analizada a través de la convergencia con otras escalas similares, con el diagnóstico clínico realizado por un especialista o mediante el análisis de perfiles y dimensiones subyacentes en diferentes muestras. Los distintos indicadores de validez han resultado, en general, satisfactorios.

c. Escala de Bienestar de Ryff. Ryff (1989a, 1989b) sugirió un modelo multidimensional de bienestar psicológico compuesto por seis dimensiones: autoaceptación, relaciones positivas con otras personas, autonomía, dominio del entorno, propósito de la vida y crecimiento personal. Es un instrumento que cuenta con un total de seis escalas y 39 ítems a los que los participantes responden utilizando un formato de respuesta con puntuaciones comprendidas entre 1 (totalmente en desacuerdo) y 6 (totalmente de acuerdo). La consistencia interna ( $\alpha$ de Cronbach) de la versión española de la Escalas de Bienestar Psicológico son: autoaceptación $\alpha=0,83$, relaciones positivas $\alpha=0,81$, autonomía $\alpha=0,83$, dominio del entorno $\alpha=0,71$, propósito en la vida $\alpha=0,83$ y crecimiento personal $\alpha=0,68$. En cuanto a la fiabilidad los valores del tercer cuarto indicador (CFI Y NNFI) cercanos a 0.90 o superiores y menores de 0.080 en el tercer indicador (SRMR) y de 0.060 en el cuarto (RMSEA), señalan un ajuste de los datos al modelos relativamente bueno.

d. Cuestionario de Autoconcepto Forma 5 (AF5) de García y Musitu. Es un instrumento diseñado para la población de los dos últimos cursos de Educación Primaria, para ESO, Bachillerato, universitarios y adultos en general, y validado para dicha población. En este instrumento el autoconcepto se presenta integrado por cinco dimensiones: académico-laboral, emocional, familiar, físico y social. Cada una de ellas es evaluada por 6 ítems, teniendo el cuestionario 30 en total. Su consistencia interna, evaluada mediante coeficiente alfa de Cronbach es más que satisfactoria, siendo de 0.81 para todo el cuestionario y para las cinco dimensiones de 0.88 , $0.73,0.76,0.74$ y 0.69 .

e. Cuestionario de Estrategias de Aprendizaje (CEA) de Jesús A. Beltrán, Luz F. Pérez e Isabel Ortega. Representa a las tres dimensiones de la mente humana, voluntad, capacidad y autonomía (querer, poder y decidir), por medio de cuatro escalas que se corresponden con el modelo de construcción del conocimiento explicado por Beltrán (1993). Este modelo comprende los siguientes procesos: sensibilización, atención, adquisición, personalización, recuperación, transferencia, evaluación y metacognición. Los valores de los coeficientes de fiabilidad, que se han obtenido son: sensibilización $\alpha=0,82$, elaboración 
$\alpha=0,87$, personalización $\alpha=0,88$, metacognición $\alpha=0,77$ y la escala total $\alpha=0,95$. La validez de constructo se ha estudiado por medio del análisis factorial de tipo exploratorio para cada una de las escalas, basándose en el método de componentes principales y el tipo de rotación varimax. Los resultados son sensibilización 0,86 , elaboración 0,92 , personalización 0,94 y metacognición 0,84 .

\section{Diseño y variables}

La investigación requiere la utilización de la prueba de Rangos señalados y pares igualados de Wilcoxon, teniendo en cuenta el objetivo e hipótesis de la investigación y basándonos en la naturaleza de las variables implicadas en el estudio, variables ordinales para grupos correlacionados. Las variables dependientes son de naturaleza ordinal y la variable independiente «aplicación del programa» define dos niveles: 1. Aplicación y 2. No aplicación. La finalidad es establecer diferencias entre dos grupos correlacionados respecto de una serie de características ordinales.

\section{Variables de Estudio}

Las variables de estudio han sido las siguientes:

a) Variable independiente:

1. Aplicación del Programa Almida.

2. No aplicación del Programa Almida.

b) Variables dependientes:

Tabla 3

Variables Dependientes

1. Intensidad sintomática de la depresión (BDI).

2. Somatizaciones.

3. Obsesiones y compulsiones.

4. Sensibilidad interpersonal.

5. Depresión.

6. Ansiedad.

7. Hostilidad.

8. Ansiedad Fóbica.

9. Ideación Paranoide.

10. Psicoticismo.

11. Severidad Global.

12. La autoaceptación.
13. La capacidad de mantener relaciones positivas con otras personas.

14 La autonomía.

15. El dominio del entorno.

16. El crecimiento personal.

17. Propósito en la vida.

18. Autoconcepto académicolaboral.

19. Autoconcepto emocional.

20. Autoconcepto familiar.

21. Autoconcepto físico.

22. Autoconcepto social.

23. Autoconcepto Global.

24. La motivación.
25. La actitud.

26. El control emocional.

27. La elaboración.

28. La organización.

29. La selección.

30. El transfer.

31. El pensamiento creativo o crítico.

32. La recuperación.

33. El plan de evaluación o planificación.

34. La regulación.

35. Puntuación total del CEA.

\section{Procedimiento}

El objetivo principal del estudio es comprobar, desde el punto de vista científico, la eficiencia del Programa Almida: Programa de
Mejora de las Competencias Sociocognitivas en Personas con Capacidades Diferentes.

Es un programa global, planificado y gestionado de manera unificada, tanto en su nivel más general como en su aplicación a personas concretas. 
La aplicación del programa se ha realizado de la manera siguiente:

1. Antes del tratamiento (Pretest). Se han aplicado el conjunto de instrumentos y pruebas, antes descritas, a la muestra seleccionada.

2. Después del tratamiento (Postest). Se han aplicado los mismos instrumentos y pruebas a la muestra seleccionada una vez habido aplicado el tratamiento.

El Tratamiento es el Programa de Mejora de las Competencias Sociocognitivas en Personas con Capacidades Diferentes: Programa Almida.

Los instrumentos, anteriormente enumerados, tratan de evaluar en las personas con discapacidad de la muestra seleccionada el nivel de:

- Depresión.

- Un amplio rango de problemas psicopatológicos.

- Bienestar psicológico.
- Autoconcepto integrado por cinco dimensiones: académico-laboral, emocional, familiar, físico y social.

- Estrategias de Aprendizaje.

El objetivo es demostrar que el Programa Almida mejora las competencias sociocognitivas en personas con capacidades diferentes, mejorando también la dimensión de los instrumentos mencionados.

\section{Análisis Estadístico}

El análisis estadístico de los datos se ha realizado con pruebas de comparación de momentos pretest y postest de las distintas variables. Se ha estudiado la comparación de las medias utilizando la prueba de Los Rangos Señalados y Pares Igualados de Wilcoxon, de acuerdo al objetivo e hipótesis de la investigación y teniendo en cuenta la naturaleza de las variables implicadas en el problema de investigación, variables ordinales para grupos correlacionados. Los análisis de la investigación han sido realizados con el paquete estadístico SPSS para Windows.

\section{Análisis Descriptivo y Estadístico de Wilcoxon}

Resultados estadísticos

Tabla 4

Datos descriptivos y estadísticos del BDI (Beck et al., 1961)

\begin{tabular}{lccccc}
\hline & Media & Varianza & $\begin{array}{c}\text { Desviación } \\
\text { Típica }\end{array}$ & Wilcoxon (Z) & $\begin{array}{c}\text { Sig. Asintót. } \\
\text { (bilateral) }\end{array}$ \\
\hline Pre & 13.60 & 95.16 & 9.75 & $-5.08(a)$ & .00 \\
Post & 7.67 & 58.49 & 7.64 & & .00 \\
\hline
\end{tabular}

Nota. (a) Basado en los rangos positivos. 
Tabla 5

Datos descriptivos y estadísticos del SCL-90 R (González Rivera, 1989)

\begin{tabular}{|c|c|c|c|c|c|c|}
\hline & & Media & Varianza & $\begin{array}{c}\text { Desviación } \\
\text { Típica }\end{array}$ & Wilcoxon (Z) & Sig. bilat \\
\hline \multirow{2}{*}{ Somatizaciones } & Pre & 1.08 & .66 & .81 & \multirow{2}{*}{$-3.74(a)$} & \multirow{2}{*}{.00} \\
\hline & Post & .81 & .49 & .70 & & \\
\hline \multirow{2}{*}{$\begin{array}{l}\text { Obsesiones y } \\
\text { compulsiones }\end{array}$} & Pre & 1.05 & .77 & .87 & \multirow{2}{*}{$-5.15(a)$} & \multirow{2}{*}{.00} \\
\hline & Post & .62 & .36 & .60 & & \\
\hline \multirow{2}{*}{$\begin{array}{l}\text { Sensibilidad } \\
\text { Interpersonal }\end{array}$} & Pre & .96 & .63 & .79 & \multirow{2}{*}{$-5.24(a)$} & \multirow{2}{*}{.00} \\
\hline & Post & .51 & .26 & .50 & & \\
\hline \multirow{2}{*}{ Depresión } & Pre & 1.11 & .76 & .87 & \multirow{2}{*}{$-5.26(a)$} & \multirow{2}{*}{.00} \\
\hline & Post & .60 & .40 & .63 & & \\
\hline \multirow{2}{*}{ Ansiedad } & Pre & .82 & .52 & .72 & \multirow{2}{*}{$-4.10(a)$} & \multirow{2}{*}{.00} \\
\hline & Post & .49 & .32 & .56 & & \\
\hline \multirow{2}{*}{ Hostilidad } & Pre & .67 & .50 & .70 & \multirow{2}{*}{$-4.46(a)$} & \multirow{2}{*}{.00} \\
\hline & Post & .32 & .24 & .48 & & \\
\hline \multirow{2}{*}{ Ansiedad Fóbica } & Pre & .45 & .32 & .56 & \multirow{2}{*}{$-3.87(a)$} & \multirow{2}{*}{.00} \\
\hline & Post & .25 & .21 & .46 & & \\
\hline \multirow{2}{*}{$\begin{array}{l}\text { Ideaciones } \\
\text { paranoides }\end{array}$} & Pre & .90 & .64 & .80 & \multirow{2}{*}{$-3.32(a)$} & \multirow{2}{*}{.00} \\
\hline & Post & .61 & .50 & .71 & & \\
\hline \multirow{2}{*}{ Psicoticismo } & Pre & .55 & .33 & .57 & \multirow{2}{*}{$-3.70(a)$} & \multirow{2}{*}{.00} \\
\hline & Post & .32 & .20 & .45 & & \\
\hline Índice de Severidad & Pre & .17 & .01 & .10 & \multirow{2}{*}{$-5.77(a)$} & \multirow{2}{*}{.00} \\
\hline General & Post & .10 & .01 & .10 & & \\
\hline
\end{tabular}

Nota. (a) Basado en los rangos positivos.

Tabla 6

Datos descriptivos y estadísticos del Nivel de Bienestar Psicológico de Ryff (1989)

\begin{tabular}{|c|c|c|c|c|c|c|}
\hline & & Media & Varianza & $\begin{array}{c}\text { Desviación } \\
\text { Típica }\end{array}$ & $\begin{array}{c}\text { Wilcoxon } \\
(\mathrm{Z})\end{array}$ & Sig. bila \\
\hline \multirow{2}{*}{ Autoaceptación } & Pre & 3.97 & 1.13 & 1.06 & \multirow{2}{*}{$-3.78(b)$} & \multirow{2}{*}{.000} \\
\hline & Post & 4.34 & 0.85 & .92 & & \\
\hline \multirow{2}{*}{ Relaciones positivas } & Pre & 4.36 & 1.28 & 1.13 & \multirow{2}{*}{$-1.04(b)$} & \multirow{2}{*}{0.29} \\
\hline & Post & 4.44 & 1.13 & 1.06 & & \\
\hline \multirow{2}{*}{ Autonomía } & Pre & 4.14 & 0.82 & .90 & \multirow{2}{*}{$-1.36(b)$} & \multirow{2}{*}{0.17} \\
\hline & Post & 4. 29 & 0.71 & .84 & & \\
\hline \multirow{2}{*}{ Dominio del entorno } & Pre & 4.17 & .74 & .86 & \multirow{2}{*}{$-3.17(b)$} & \multirow{2}{*}{0.02} \\
\hline & Post & 4.47 & .67 & .82 & & \\
\hline \multirow{2}{*}{ Crecimiento personal } & Pre & 4.41 & .66 & .81 & \multirow{2}{*}{$-2.34(b)$} & \multirow{2}{*}{0.02} \\
\hline & Post & 4.56 & .50 & .71 & & \\
\hline \multirow{2}{*}{ Propósito en la vida } & Pre & 4.29 & 1.14 & 1.06 & \multirow{2}{*}{$-3.41(b)$} & \multirow{2}{*}{0.01} \\
\hline & Post & 4.64 & .88 & .94 & & \\
\hline
\end{tabular}

Nota. (b) Basado en los rangos negativos. 
Tabla 7

Datos descriptivos y estadísticos del Autoconcepto Forma 5 (AF5), García y Musitu (2001)

\begin{tabular}{|c|c|c|c|c|c|c|}
\hline & & Media & Varianza & $\begin{array}{l}\text { Desviación } \\
\text { Típica }\end{array}$ & $\begin{array}{c}\text { Wilcoxon } \\
(\mathrm{Z})\end{array}$ & Sig. bilat \\
\hline \multirow{2}{*}{$\begin{array}{l}\text { Autoconcepto Académico } \\
\text { Laboral }\end{array}$} & Pre & 72.79 & 277.60 & 16.66 & \multirow{2}{*}{$2.25(b)$} & \multirow{2}{*}{0.02} \\
\hline & Post & 77.67 & 191.19 & 13.82 & & \\
\hline \multirow{2}{*}{ Autoconcepto Emocional } & Pre & 42.44 & 498.38 & 22.32 & \multirow{2}{*}{$-1.67(a)$} & \multirow{2}{*}{0.09} \\
\hline & Post & 38.54 & 484.24 & 22.01 & & \\
\hline \multirow{2}{*}{ Autoconcepto Familiar } & Pre & 76.26 & 328.68 & 18.13 & \multirow{2}{*}{$-.94(b)$} & \multirow{2}{*}{0.34} \\
\hline & Post & 79.10 & 284.38 & 16.86 & & \\
\hline \multirow{2}{*}{ Autoconcepto Físico } & Pre & 51.09 & 429.28 & 20.72 & \multirow{2}{*}{$-2.34(b)$} & \multirow{2}{*}{0.02} \\
\hline & Post & 55.94 & 386.09 & 19.65 & & \\
\hline \multirow{2}{*}{ Autoconcepto Social } & Pre & 66.80 & 422.08 & 20.54 & \multirow{2}{*}{$-2.27(b)$} & \multirow{2}{*}{0.02} \\
\hline & Post & 71.86 & 303.27 & 17.41 & & \\
\hline \multirow{2}{*}{ Autoconcepto Global } & Pre & 61.88 & 135.6 & 11.64 & \multirow{2}{*}{$-1.68(b)$} & \multirow{2}{*}{0.09} \\
\hline & Post & 64.62 & 87.69 & 9.36 & & \\
\hline
\end{tabular}

Nota. (a) Basado en los rangos positivos. (b) Basado en los rangos negativos.

Tabla 8

Datos descriptivos y estadísticos del Cuestionario de Estrategias de Aprendizaje (CEA) de Jesús A. Beltrán, Luz F. Pérez e Isabel Ortega (2006)

\begin{tabular}{|c|c|c|c|c|c|c|}
\hline & & Media & Varianza & $\begin{array}{c}\text { Desviación } \\
\text { Típica }\end{array}$ & $\begin{array}{c}\text { Wilcoxon } \\
(\mathrm{Z}) \\
\end{array}$ & Sig. bilat \\
\hline \multirow{2}{*}{ Motivación } & Pre & 2.49 & .50 & .70 & \multirow{2}{*}{$-3.25(b)$} & \multirow{2}{*}{0.001} \\
\hline & Post & 2.68 & .50 & .70 & & \\
\hline \multirow{2}{*}{ Actitud } & Pre & 2.58 & 1.11 & 1.05 & \multirow{2}{*}{$-2.42(b)$} & \multirow{2}{*}{0.01} \\
\hline & Post & 2.85 & 1.01 & 1.00 & & \\
\hline \multirow{2}{*}{ Control emocional } & Pre & 2.32 & .69 & .83 & \multirow{2}{*}{$-1.01(b)$} & \multirow{2}{*}{0.3} \\
\hline & Post & 2.38 & .72 & .85 & & \\
\hline \multirow{2}{*}{ Elaboración } & Pre & 2.30 & .67 & .82 & \multirow{2}{*}{$-1.54(b)$} & \multirow{2}{*}{0.12} \\
\hline & Post & 2.39 & .69 & .83 & & \\
\hline \multirow{2}{*}{ Organización } & Pre & 2.31 & 1.04 & 1.02 & \multirow{2}{*}{$-1.13(b)$} & \multirow{2}{*}{0.25} \\
\hline & Post & 2.43 & 1.35 & 1.16 & & \\
\hline \multirow{2}{*}{ Selección } & Pre & 2.18 & .84 & .92 & \multirow{2}{*}{$-2.19(b)$} & \multirow{2}{*}{0.02} \\
\hline & Post & 2.40 & .95 & .97 & & \\
\hline \multirow{2}{*}{ Transferencia } & Pre & 2.01 & .77 & .88 & \multirow{2}{*}{$-2.04(b)$} & \multirow{2}{*}{0.04} \\
\hline & Post & 2.19 & .83 & .91 & & \\
\hline \multirow{2}{*}{ Pensamiento creativo } & Pre & 2.03 & .54 & .73 & \multirow{2}{*}{$-2.43(b)$} & \multirow{2}{*}{0.01} \\
\hline & Post & 2.16 & .53 & .73 & & \\
\hline \multirow{2}{*}{ Recuperación } & Pre & 2.13 & .78 & .88 & \multirow{2}{*}{$-.51(b)$} & \multirow{2}{*}{0.61} \\
\hline & Post & 2.19 & .83 & .91 & & \\
\hline \multirow{2}{*}{ Planificación, Evaluación } & Pre & 2.15 & .74 & .86 & \multirow{2}{*}{$-2.17(b)$} & \multirow{2}{*}{0.03} \\
\hline & Post & 2.34 & .83 & .91 & & \\
\hline \multirow{2}{*}{ Regulación } & Pre & 3.11 & .53 & .73 & \multirow{2}{*}{$-0.17(b)$} & \multirow{2}{*}{0.86} \\
\hline & Post & 3.08 & .74 & .86 & & \\
\hline Total & Pre & 2.33 & .38 & .62 & & 001 \\
\hline lotal & Post & 2.46 & .43 & .65 & -2.51 (D) & 0.01 \\
\hline
\end{tabular}

Nota. (b) Basado en los rangos negativos. 
Los resultados de la prueba de Wilcoxon nos informan de la existencia de diferencias estadísticamente significativas en los rangos del pretest y del postest. La tabla de rangos que se muestra nos sirve para contrastar la información aportada por la prueba de Wilcoxon.

VARIABLE INDEPENDIENTE: Aplicación del Programa (pretest/ postest).

VARIABLE DEPENDIENTE: Distintas variables evaluadas.

\section{Resultados}

Resultados de las hipótesis principales

Tabla 9

Resultados Hipótesis principales
Hipótesis nula: No existen diferencias estadísticamente significativas entre el pretest y el postest.

Hipótesis alterna: Existen diferencias estadísticamente significativas entre el pretest y el postest.

El valor de contraste que utilizaremos $\alpha$ será igual a .05.

Los contrastes se realizan para cada una de las variables, relacionando su puntuación en el pretest y en el postest.

\begin{tabular}{|c|c|c|c|c|c|}
\hline $\begin{array}{c}\text { Instrumento } \\
\text { o Prueba }\end{array}$ & Variable & $\begin{array}{c}\text { Programa } \\
\text { Almida }\end{array}$ & Mejora & $\begin{array}{l}\text { No Difer. } \\
\text { Signif. }\end{array}$ & Empeora \\
\hline 1. BDI & Depresión & Interv. & $x$ & & \\
\hline \multirow{10}{*}{ 2. SCL-90-R } & Somatizaciones & Interv. & $X$ & & \\
\hline & $\begin{array}{l}\text { Obsesiones } \\
\text { Compulsiones }\end{array}$ & Interv. & $x$ & & \\
\hline & $\begin{array}{l}\text { Sensibilidad } \\
\text { Interpersonal }\end{array}$ & Interv. & $x$ & & \\
\hline & Depresión & Interv. & $\mathrm{X}$ & & \\
\hline & Ansiedad & Interv. & $X$ & & \\
\hline & Hostilidad & Interv. & $X$ & & \\
\hline & Ansiedad Fóbica & Interv. & $\mathrm{X}$ & & \\
\hline & Ideación Paranoide & Interv. & $\mathrm{X}$ & & \\
\hline & Psicoticismo & Interv. & $\mathrm{X}$ & & \\
\hline & Severidad Global & Interv. & $\mathrm{X}$ & & \\
\hline \multirow{6}{*}{$\begin{array}{l}\text { 3. Escala de Bienestar } \\
\text { Psicológico de Ryff. }\end{array}$} & Autoaceptación & Interv. & $\mathrm{X}$ & & \\
\hline & Relaciones Positivas & Interv. & & $X$ & \\
\hline & Autonomía & Interv. & & $\mathrm{X}$ & \\
\hline & Dominio del Entorno & Interv. & $X$ & & \\
\hline & Crecimiento Personal & Interv. & $\mathrm{X}$ & & \\
\hline & Propósito en la Vida & Interv. & $\mathrm{X}$ & & \\
\hline \multirow{6}{*}{ 4. Cuestionario AF 5} & $\begin{array}{l}\text { Autoc. Académico } \\
\text { laboral }\end{array}$ & Interv. & $x$ & & \\
\hline & Autoc. Emocional & Interv. & & $x$ & * \\
\hline & Autoc. Familiar & Interv. & & $\mathrm{X}$ & \\
\hline & Autoc. Fisico & Interv. & $\mathrm{X}$ & & \\
\hline & Autoc. Social & Interv. & $X$ & & \\
\hline & Autoc. Global & Interv. & & $X$ & \\
\hline
\end{tabular}




\begin{tabular}{|c|c|c|c|c|c|}
\hline $\begin{array}{c}\text { Instrumento } \\
\text { o Prueba }\end{array}$ & Variable & $\begin{array}{c}\text { Programa } \\
\text { Almida }\end{array}$ & Mejora & $\begin{array}{c}\text { No Difer. } \\
\text { Signif. }\end{array}$ & Empeora \\
\hline \multirow{12}{*}{ 5. CEA } & Motivación & Interv. & $\mathrm{X}$ & & \\
\hline & Actitud & Interv. & $\mathrm{x}$ & & \\
\hline & Control Emocional & Interv. & & $\mathrm{X}$ & \\
\hline & Elaboración & Interv. & & $\mathrm{X}$ & \\
\hline & Organización & Interv. & & $\mathrm{X}$ & \\
\hline & Selección & Interv. & $x$ & & \\
\hline & Transfer & Interv. & $\mathrm{x}$ & & \\
\hline & Pensamiento Creativo & Interv. & $\mathrm{x}$ & & \\
\hline & Recuperación & Interv. & & $\mathrm{X}$ & \\
\hline & $\begin{array}{l}\text { Plan Eval. o } \\
\text { Planificación }\end{array}$ & Interv. & $x$ & & \\
\hline & Regulación & Interv. & & $\mathrm{X}$ & \\
\hline & Total CEA & Interv. & $\mathrm{X}$ & & \\
\hline
\end{tabular}

Además, de manera global, los resultados nos reflejan que de 35 variables dependientes estudiadas, 25 de ellas mejoran su puntuación e interpretación por la aplicación del Programa Almida, y 10 no tienen diferencias significativas en los resultados en pretest y postest.

Hay una variable que tiene un resultado adverso. La variable Autoconcepto Emocional se comporta después del tratamiento con un resultado que la muestra obtiene peor resultado en el postest que en el pretest, pero las diferencias no son significativas.

En definitiva, el Programa Almida mejora en el $71.42 \%$ de las variables estudiadas, de igual modo, produce un efecto muy positivo y significativo en las personas con capacidades diferentes.

\section{Conclusiones}

Los estudios de investigación con personas con discapacidad han tenido suscitado un interés general y tienen ya una profunda tradición de investigaciones y publicaciones. Sin embargo, a pesar del volumen de estas aportaciones, la incorporación a la práctica formativa de las innovaciones y mejoras propuestas desde la investigación resulta aún muy escasa. Por esta razón en el desarrollo de este estudio se ha intentado buscar un espacio de encuentro entre los fundamentos teóricos de la orientación y la tutoría y la aplicación práctica.
Esta investigación se ha planteado como una intervención en el contexto, de manera que se han podido integrar los conocimientos obtenidos a partir de la construcción de su marco teórico referencial y la actuación profesional en las diferentes fases del trabajo, con el objetivo de desarrollar de la mejor manera el Programa de Mejora de las Competencias Sociocognitivas en Personas con Capacidades Diferentes.

En ningún momento se puede valorar este estudio como un "resultado terminado», sino sólo como un punto de partida que en el contexto de la formación, orientación e inserción de las personas con capacidades diferentes, podría servir de marco de referencia para futuras reflexiones y el desarrollo de nuevas investigaciones por parte de especialistas interesados en la temáticas de la orientación de las personas con discapacidad.

El Programa Almida se ha inspirado principalmente en el Paradigma Cognitivo, en la orientación psicopedagógica y el aprendizaje significativo. En personas con capacidades diferentes la orientación es una intervención psicopedagógica cuya finalidad es la integración social y laboral.

\section{Discusión}

En este estudio se ha conseguido el propósito de la investigación; comprobar la eficiencia del Programa Almida como un Programa 
de Mejora de las Competencias Sociocognitivas en personas con Capacidades Diferentes, a partir de la de un marco referencial específico que permite intervenir en este contexto.

Además, se han alcanzado los siguientes objetivos:

1. Comprobar, desde el punto de vista científico, la eficiencia del Programa Almida como conjunto de actuaciones de orientación social y laboral y asesoramientos para la consecución de competencias sociales, intelectuales y cognitivas, que pretenden mejorar la autonomía personal, la responsabilidad sobre el proceso de aprendizaje y la adquisición de competencias que ayuden a superar retos y desafíos de la vida.

2. Los resultados reflejan que de 35 variables dependientes estudiadas, 25 de ellas han mejorado su puntuación e interpretación por la aplicación del Programa Almida, y 10 no han tenido diferencias significativas en los resultados en pretest y postest. Se ha encontrado una variable que ha tenido un resultado adverso: la variable Autoconcepto Emocional se ha comportado después del tratamiento con un resultado que la muestra obtiene peor resultado en el postest que en el pretest, pero las diferencias no han sido significativas entre los dos momentos.
En definitiva, el Programa Almida ha mejorado en el $71.42 \%$ de las variables estudiadas, por tanto, produce un efecto muy positivo en las personas con capacidades diferentes.

Por tanto, el Programa de Mejora de las Competencias Sociocognitivas es un conjunto de actuaciones de orientación laboral y asesoramientos para la ocupación laboral, que mejora la sintomatología depresiva de las personas con capacidades diferentes, les reconforta y ayuda a superar problemas psicopatológicos como somatizaciones, obsesiones, ansiedad, etc., incrementa positivamente el bienestar psicológico en sus dimensiones como la autoaceptación, el dominio del entorno, el propósito en la vida, etc., hace progresar el autoconcepto académico-laboral, el físico y el social, además de ayudar a adquirir y desarrollar estrategias y procesos de aprendizaje como la motivación, la actitud, selección, transfer, pensamiento creativo y planificación-evaluación.

Finalmente, este proceso hace que las personas con capacidades diferentes cambien en varios sentidos: mejorando las competencias para afrontar sus expectativas, mejorando en la percepción misma (autoconcepto) y mejorando en la autovaloración que se hace de sí misma (autoestima). En este sentido, el concepto de Empleabilidad favorece social y personalmente a la persona con capacidades diferentes. 


\section{Referencias}

Beck A. T. y Beck R. W. (1972). Screening depressed patients in family practice. A rapid technic. Postgrad Med, 52, 81-85.

Beck A. T., Rial W. Y. y Rickels K. (1974). Short form of Depression Inventory (Cross-validation). Psychological Reports, 34, 1184-1186.

Beck A. T., Steer R. A. y Garbin M.G. (1988). Psychometric properties of the Beck Depression Inventory. Twenty-five years of evaluation. Clin Psychol Rev, 8, 77-100.

Beck A. T., Steer R. A. y Brown G.K. (1996). Manual for the Beck Depression Inventory-II. San Antonio, TX: Psychological Corporation.

Beltrán, J. A. (1993). Procesos, estrategias y técnicas de aprendizaje [Processes, learning strategies and techniques]. Madrid, España: Síntesis.

Beltrán, J. A. (1996). Estrategias de aprendizaje [Learning strategies]. En J. A. Beltrán y C. Genovard (Eds.), Psicología de la Instrucción I: variables y procesos básicos [Instructional Psychology I: variables and basic processes]. Madrid, España: Síntesis.

Beltrán, J. A. (2001). El aprendizaje significativo: naturaleza, estructura y función [Meaningful learning: nature, structure and function]. Cuadernos de Pensamiento, 14, 133-151

Beltrán, J. A. y Bueno Álvarez, J. A. (1987). Psicología de la Educación [Educational Psychology]. Madrid, España: Eudema.

Beltrán, J. y Bueno Álvarez, J. A. (1995). Psicología de la Educación [Educational Psychology]. Madrid, España: Marcombo.

Beltrán, J. A. y Genovard, C. (1999). Psicología de la Instrucción. Áreas curriculares [Instructional Psychology. Curricular areas]. Madrid, España: Síntesis.

Beltrán, J. A., Pérez, L. F. y Ortega, M. I. (2006). Cuestionario de Estrategias de Aprendizaje (CEA) [Learning Strategies Questionnaire]. Madrid, España: TEA.

Cáritas Española (1999). ¿Cómo desarrollar la Empleabilidad? [How to develop employability?]. Madrid, España: Cuadernos para la Inserción.

Ceniceros Estévez, J. C. y Oteo Antuñano, E. (2003). Orientación sociolaboral basada en itinerarios.
Una propuesta metodológica para la intervención con personas en riesgo de exclusión [Guidance based socio itineraries. A proposed methodology for intervention with those at risk of exclusion]. Madrid, España: Fundación Tomillo.

Derogatis L. R., Lipman, R. y Covi L. (1973). SCL-90: An outpatient psychiatric rating scale preliminary report. Psychopharmacology Bulletin, 9, 13-28.

Derogatis, L. R. Rickels, K. y Rock, A. (1976). The SCL-90 and the MMPI: a step in the validation of a new self-report scale. The British Journal of Psychiatry, 128, 280-289.

García, F. y Musitu, G. (2001). AF5. Autoconcepto Forma 5 [AF5. Self Form 5]. Madrid, España: TEA.

García Pastor, C. y Álvarez Rojo, V. (1995). La orientación profesional de jóvenes con necesidades educativas especiales: las funciones del departamento de orientación y los nuevos roles de los orientadores [Career guidance for young people with special educational needs: the guidance department functions and new roles of counselors]. En J. Fernández Sierra (Ed.), El trabajo docente y psicopedagógico en Educación Secundaria [The teachers work in secondary education and educational psychology]. Archidona, M. A.: Aljibe, pp. 329-343.

Gardner, H. (1995). Inteligencias Múltiples. La teoría en la práctica [Multiple Intelligences. The theory in practice]. Barcelona, España: Paidós.

Gardner, H. (1997). Estructuras de la mente: La teoría de las Inteligencia Múltiples [Frames of Mind: The Theory of Multiple Intelligence]. Bogotá, Colombia: Fondo de Cultura Económica.

Gardner, H. (1999). Intelligence Reframed: Multiple Intelligences for the 21st Century. New York: Basic Books.

Gardner, H. (2007). Five Minds for the Future. La teoría en la práctica [Five Minds for the Future. The theory in practice]. Boston (US): Harvard business school press.

González de Rivera, J. L., De las Cuevas, C., Rodríguez, M. y Rodríguez, F. (2002). Cuestionario de 90 sintomas $S C L-90-R$ de Derogatis, L. Adaptación española [90 Symptoms Questionnaire SCL-90-R of Derogatis, L. Spanish adaptation]. Madrid, España: TEA.

Nickerson, R. S., Perkins, D. N. y Smith, E. E. (1985) Enseñar a Pensar [Teaching Thinking]. Barcelona, España: Paidós. 
Ryff, C. (1989a). Beyond Ponce de Leon and life satisfaction: New directions in ques of successful aging. International Journal of Behavioral Development, 12, 35-55.

Ryff, C. (1989b). Happines is everything, or is it? Explorations on the meaning of psychological well being. Journal of Personality and Social Psychology, 57, 1069-1081.

Ryff, C., y Keyes, C. (1995). The structure of psychological well-being revisited. Journal of Personality and Social Psychology, 69, 719-727.

Ryff, C. y Singer, B. (1998). The contours of positive health. Psychological Inquiry, 9(11), 1-28.

Ryff, C., y Singer, B. (2002). From social structure to biology. In C. Snyder y A. López (Eds.), Hand- book of positive psychology (pp. 63-73). Londres: Oxford University Press.

Sternberg, R. J. (1986). Las capacidades humanas. Un enfoque desde el procesamiento de la información [Human capabilities. An approach from the information processing]. Barcelona, España: Labor S.A.

Sternberg, R. J. y Spear-Swerling, L. (1996). Enseñar a Pensar [Teaching Thinking]. Madrid, España: Aula XXI, Santillana.

Sternberg, R. J. (1997). Inteligencia Exitosa [Successful Intelligence]. Barcelona, España: Paidós.

Sternberg, R. J. (1999). The theory of successful intelligence. Review of General Psychology, 3, 292-316. 